\title{
Erratum to: Effect of Aripiprazole on Verbal Memory and Fluency in Schizophrenic Patients
}

\author{
Results from the ESCAPE Study
}

\author{
Chris Bervoets - Manuel Morrens - Kristof Vansteelandt • Frank Kok • \\ Annick de Patoul - Veronique Halkin • Didier Pitsi - Eric Constant • \\ Joseph Peuskens $\cdot$ Bernard Sabbe
}

Published online: 16 October 2012

(c) Springer International Publishing Switzerland 2012

\section{Erratum to: CNS Drugs \\ DOI 10.1007/s40263-012-0003-4}

A Published-Ahead-of-Print version of this article was made available online at http://adisonline.com/cnsdrugs/ toc/publishahead on 24 September 2012. Errors were subsequently identified in the article, and the following corrections should be noted:

The online version of the original article can be found under doi:10.1007/s40263-012-0003-4.

C. Bervoets $(\square)$

Psychiatric Hospital Onze Lieve Vrouw,

Koning Albert 1 laan 8, 8200 Brugge, Belgium

e-mail: chris.bervoets@ua.ac.be

C. Bervoets · M. Morrens · B. Sabbe

Collaborative Antwerp Psychiatric Research Institute,

Antwerp, Belgium

\section{Morrens}

Psychiatric Centre Broeders Alexianen, Boechout, Belgium

K. Vansteelandt $\cdot$ J. Peuskens

University Psychiatric Centre KU Leuven, Kortenberg, Belgium

F. Kok

MSOURCE, Vianen, The Netherlands

A. de Patoul · V. Halkin · D. Pitsi

Bristol-Myers Squibb SA, Braine-l'Alleud, Belgium

E. Constant

Cliniques Universitaires Saint Luc, Brussels, Belgium

B. Sabbe

Psychiatric Hospital Sint Norbertus, Duffel, Belgium
Table 2, 'intolerability' row: The cell entry in column 2, which previously read: "9 (2.9)" has been moved to the 'other' row immediately above. The intolerability row should be empty.

In Fig. 1, A single asterisk, to indicate a $p$-value of less than 0.0001 compared with baseline, has been added to two positions, at week 4 and week 12, for the California Verbal

Table 2 Primary reason for initiating or switching to aripiprazole

\begin{tabular}{lc}
\hline Category & $\mathrm{n}(\%)$ \\
\hline No antipsychotic in past & $11(3.1)$ \\
Antipsychotic in past but not currently & $35(9.7)$ \\
Currently taking antipsychotic & $315(87.3)$ \\
Lack of efficacy & \\
Positive symptoms & $78(24.7)$ \\
Negative symptoms & $70(22.2)$ \\
Other & $9(2.9)$ \\
Intolerability & \\
Somnolence & $23(7.3)$ \\
Weight gain & $57(18.0)$ \\
Prolactin elevation & $8(2.5)$ \\
Akathisia & $3(1.0)$ \\
Other EPS & $8(2.5)$ \\
Lipid abnormality & $3(1.0)$ \\
Glucose abnormality & $4(1.3)$ \\
Other & $9(2.9)$ \\
Other & \\
Cognition & $11(3.5)$ \\
Energy & $25(7.9)$ \\
Mood & $5(1.6)$ \\
Other & $3(1.0)$ \\
No reason reported & $34(10.5)$ \\
\hline$E P S$ extrapyramidal sympto
\end{tabular}

EPS extrapyramidal symptoms 


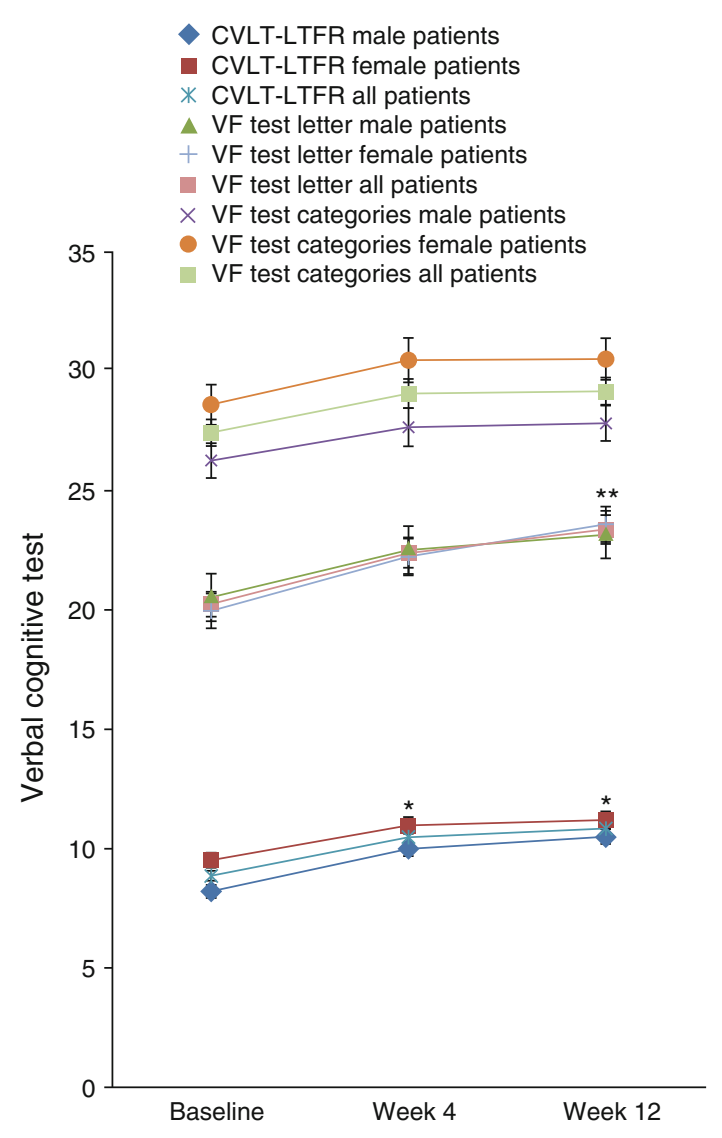

Fig. 1 Effectiveness of aripiprazole on verbal cognitive function over visits. CVLT California Verbal Learning Test, LTFR long term free recall, $V F$ verbal fluency; ${ }^{*} p<0.0001, * * p=0.0289$

Learning Test-long term free recall data. A double asterisk, to indicate a $p$-value of 0.0289 , has been inserted at week $\underline{12}$ for the verbal fluency letter data. 